\title{
Primary Nasopharyngeal Tuberculosis Combined with Tuberculous Otomastoiditis and Facial Nerve Palsy
}

\author{
Hee Young Choi, ${ }^{1}$ Ji Hye Jang, ${ }^{2}$ Kyung Mi Lee, ${ }^{2}$ Woo Suk Choi, ${ }^{2}$ Sang Hoon Kim, ${ }^{3}$ Seung Geun \\ Yeo, and Eui Jong $\operatorname{Kim}^{1,2,}$ \\ ${ }^{1}$ Department of Radiology, Graduate School, Kyung Hee University, Seoul, Korea \\ ${ }^{2}$ Department of Radiology, College of Medicine, Kyung Hee University Hospital, Kyung Hee University, Seoul, Korea \\ ${ }^{3}$ Department of Otolaryngology, College of Medicine, Kyung Hee University Hospital, Kyung Hee University, Seoul, Korea \\ *Corresponding author: Eui Jong Kim, Department of Radiology, College of Medicine, Kyung Hee University Hospital, Kyung Hee University, P. O. Box: 130-701, Seoul, Korea. Tel: \\ +822-9588611, Fax:+822-9680787, E-mail: euijkim@hanmail.net
}

Received 2015 June 22; Revised 2015 August 3; Accepted 2015 September 27.

\section{Abstract}

Primary nasopharyngeal tuberculosis (TB) without pulmonary involvement is rare, even in endemic areas. Herein, we present a rare complication of primary nasopharyngeal TB accompanied with tuberculous otomastoiditis (TOM) and ipsilateral facial nerve palsy, in a 24-year-old female patient, with computed tomography and magnetic resonance imagery findings.

Keywords: Facial Nerve, Mastoiditis, Nasopharyngeal Diseases, Paralysis, Tuberculosis

\section{Introduction}

Upper respiratory tuberculosis (TB) is a rare extrapulmonary disease, even in endemic areas (1). The nasopharynx is the least common site for TB of the upper respiratory tract (2-4). Until now, only 16 cases of primary nasopharyngeal TB have been reported in PubMed, beginning from 1967. The number of the literature reviews, including case reports about primary TB of the nasopharynx, by the search terms (primary tuberculosis, nasopharynx) was 26. Most cases of nasopharyngeal TB usually occur with combined active pulmonary TB or systemic infection, spreading via hematogenous or lymphatic systems (3). Conversely, primary nasopharyngeal TB, without pulmonary involvement, is an extremely rare disease, with very few reported cases in recent years $(1,4)$. In addition, $\mathrm{TB}$ of the middle ear cavity is also a rare extra-pulmonary manifestation (5). The incidence of tuberculous otomastoiditis (TOM) comprises $0.04-0.9 \%$ of all cases of chronic otitis media (6).

\section{Case Presentation}

A 24-year-old Asian female patient was admitted to our hospital, with left ear fullness and otorrhea. At an outpatient clinic, she was previously diagnosed with otitis media with effusion (OME) that was unresponsive to a three-week course of antibiotics. Otoscopic examination revealed perforation of the tympanic membrane and profound amber-colored discharge, behind the ear- drum (Figure 1A). On physical examination, no palpable neck lymph nodes were noted. Routine hematologic examination revealed normal erythrocyte sedimentation rate (ESR) and C-reactive protein (CRP) levels. Initial ear culture studies did not identify any pathogens. After one week of further antibiotic medication, she underwent ventilation tube (V-tube) insertion to maintain aeration of the middle ear and prevent fluid reaccumulation.

However, the presence of purulent discharge from the V-tube was again noted on the third follow-up visit (Figure $1 \mathrm{~B})$. At this visit, the patient also presented with newonset ipsilateral facial nerve palsy. Facial paralysis was identified as grade IV, using the House-Brackmann grading system, due to presence of incomplete eye closure and mouth drooping, with maximum effort. She was then hospitalized for further evaluation.

After hospitalization, the patient underwent a flexible nasopharyngoscopy, which revealed a polypoid mass, with severe inflammation, in the roof of the nasopharyngeal wall. Extensive purulent granulation tissue was also noted, along the left posterolateral nasopharyngeal wall (Figure 2). A biopsy was taken from the polypoid mass of the nasopharyngeal roof. The chest radiography was normal (Figure 3). The patient had no underlying disease, no history of pulmonary TB or contact with TB patients. Because the patient had no pulmonary symptoms and the chest radiography was normal, we excluded primary pulmonary TB. We did not perform sputum acid-fast bacilli (AFB) staining.

Copyright (C) 2016, Tehran University of Medical Sciences and Iranian Society of Radiology. This is an open-access article distributed under the terms of the Creative Commons Attribution-NonCommercial 4.0 International License (http://creativecommons.org/licenses/by-nc/4.0/) which permits copy and redistribute the material just in noncommercial usages, provided the original work is properly cited. 

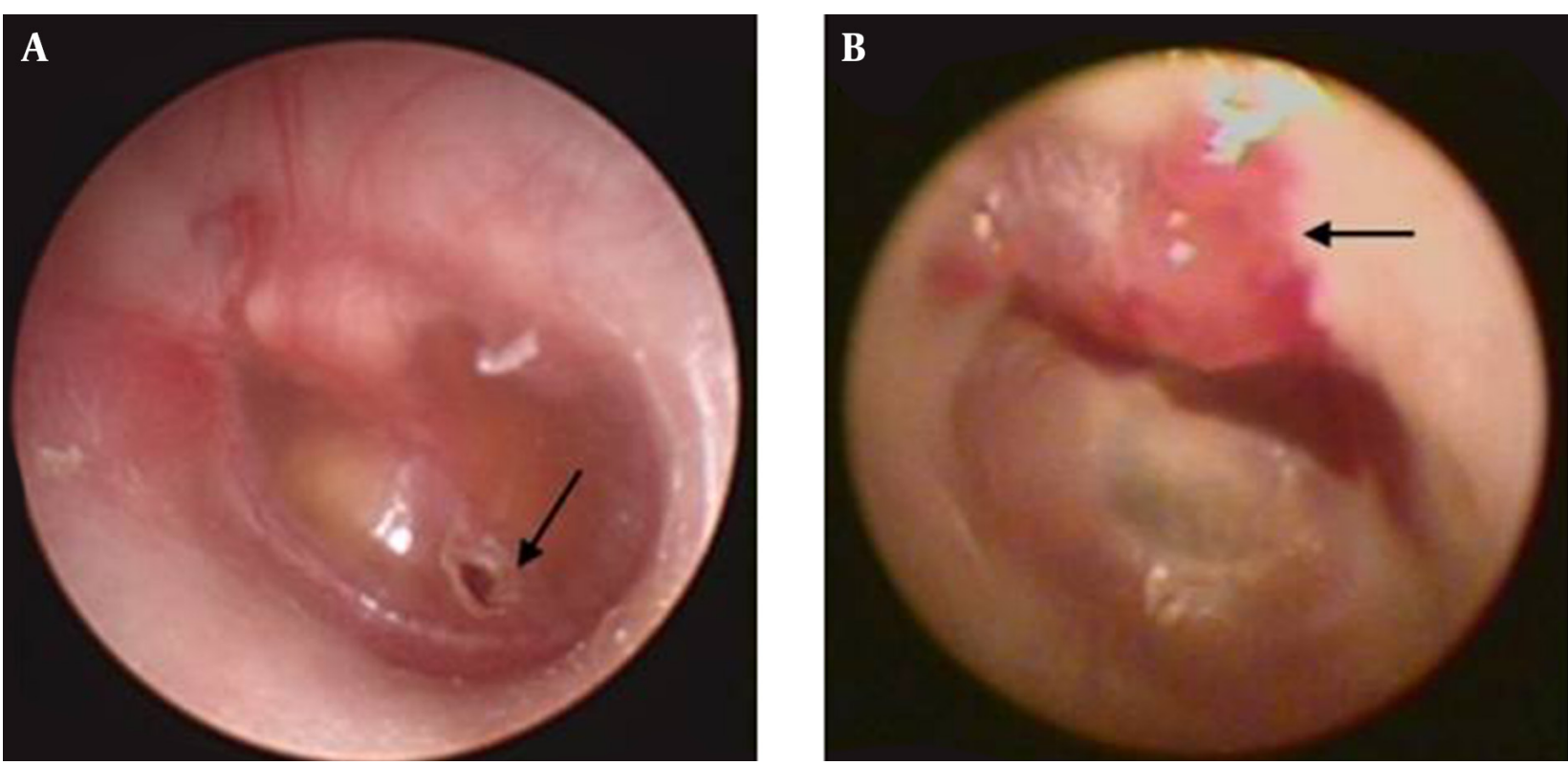

Figure 1. A 24-year-old female with ear fullness, tympanic perforation and otorrhea. Otoscopic examination upon presentation and 1 week after therapy. Initial otoscopic examination. A, Perforated tympanic membrane (arrow) with profound amber-colored discharge behind the membrane. Otoscopic examination at 1 week after tympanostomy tube (V-tube) insertion; B, Persistent purulent discharge and polypoid mass (arrow), at the superior wall of the external auditory canal.

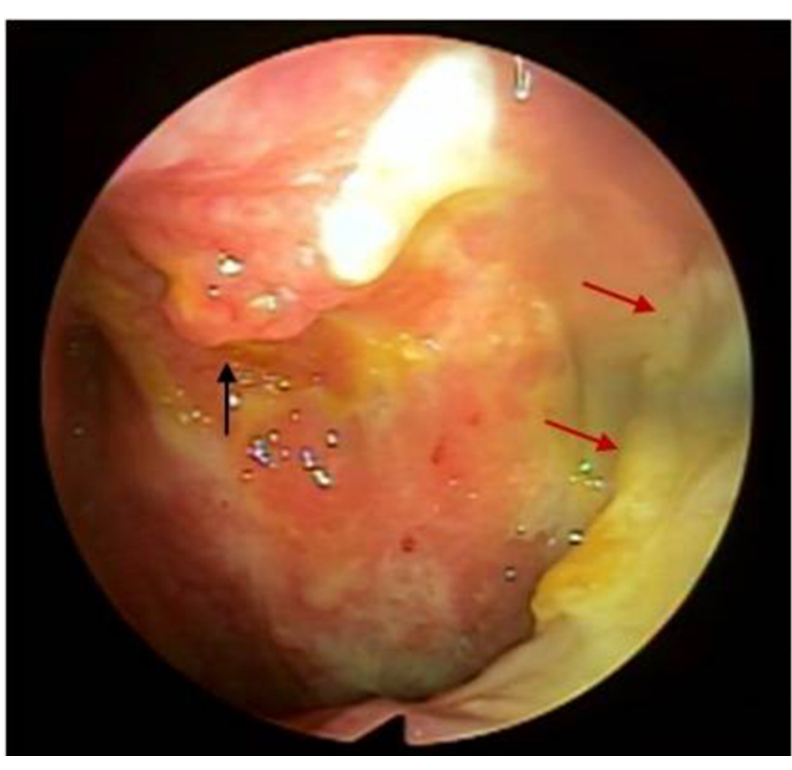

Figure 2. Fiberscopic examination of nasopharynx. A polypoid mass, with purulent discharge (black arrow) at posterior roof of nasopharyngea wall, as well as yellowish granulation tissue at left posterolateral wall of nasopharynx obliterating the Eustachian tube opening (red arrows) are visible.

The computed tomography(CT) examinations were performed using a 64 channel multi-detector computed tomography (MDCT) (Brilliance 64, Philips Medical System, Cleveland, OH, USA) or 16 channel MDCT (Light Speed, GE Healthcare, Milwaukee, WI, USA) with intravenous administration of contrastmedium(iohexol,Omnipaque $350 \mathrm{mg}$, GE Healthcare, Milwaukee, WI, USA). Magnetic resonance

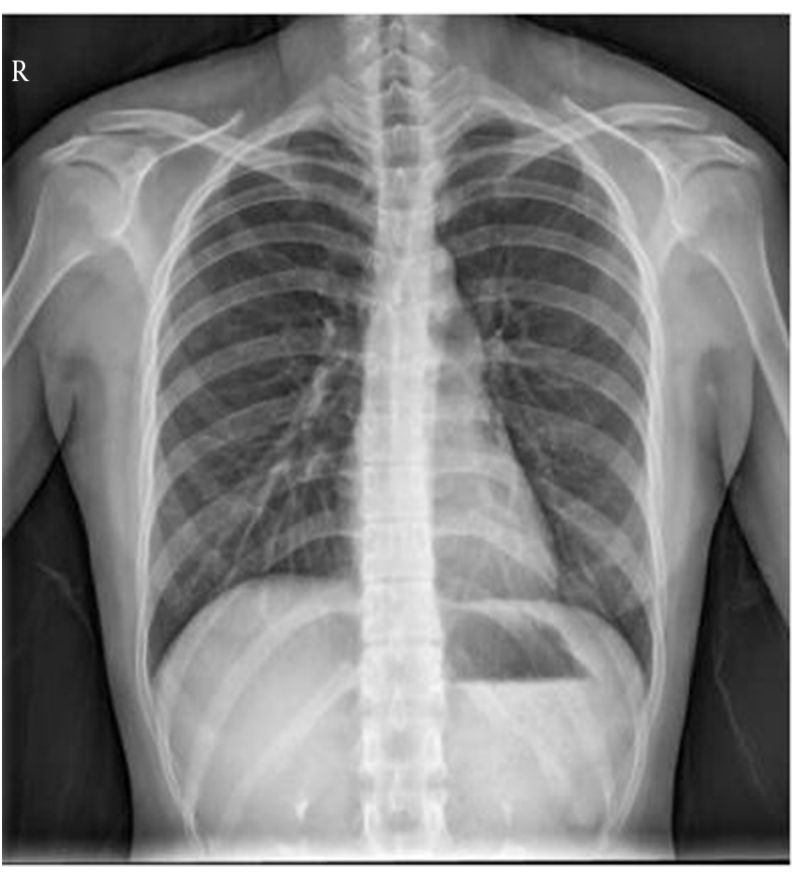

Figure 3. Chest radiography, No active lung lesion is visible.

(MR) examinations were performed using a 3.0-T MR system (Intera Achieva 3.0-T; Philips Medical Systems, Best, the Netherlands) with intravenous administration of a total volume of $15 \mathrm{~mL}$ contrast medium (gadoteridol, ProHance, Bracco Diagnostics Inc., Princeton, NJ, USA). Contrast-enhanced CT and MR of the paranasal sinuses showed a polypoid mass, with necrotic foci, at the roof of 
the nasopharyngeal wall (Figure $4 \mathrm{~A}$ and $\mathrm{B}$ ). It also revealed diffuse mucosal enhancement and low attenuated polypoid masses, obliterating the left torus tubarius and pharyngeal opening of the Eustachian tube (Figure 5A and B). Unenhanced temporal CT showed fluid filled middle ear cavity, mastoid antrum and mastoid air cells, without sclerotic changes (Figure 6). Contrast-enhanced temporal MRI showed avid enhancement at the canalicular, labyrinthine, anterior genu, tympanic and mastoid segments of the left facial nerve, which confirmed left facial neuritis (Figure 7).
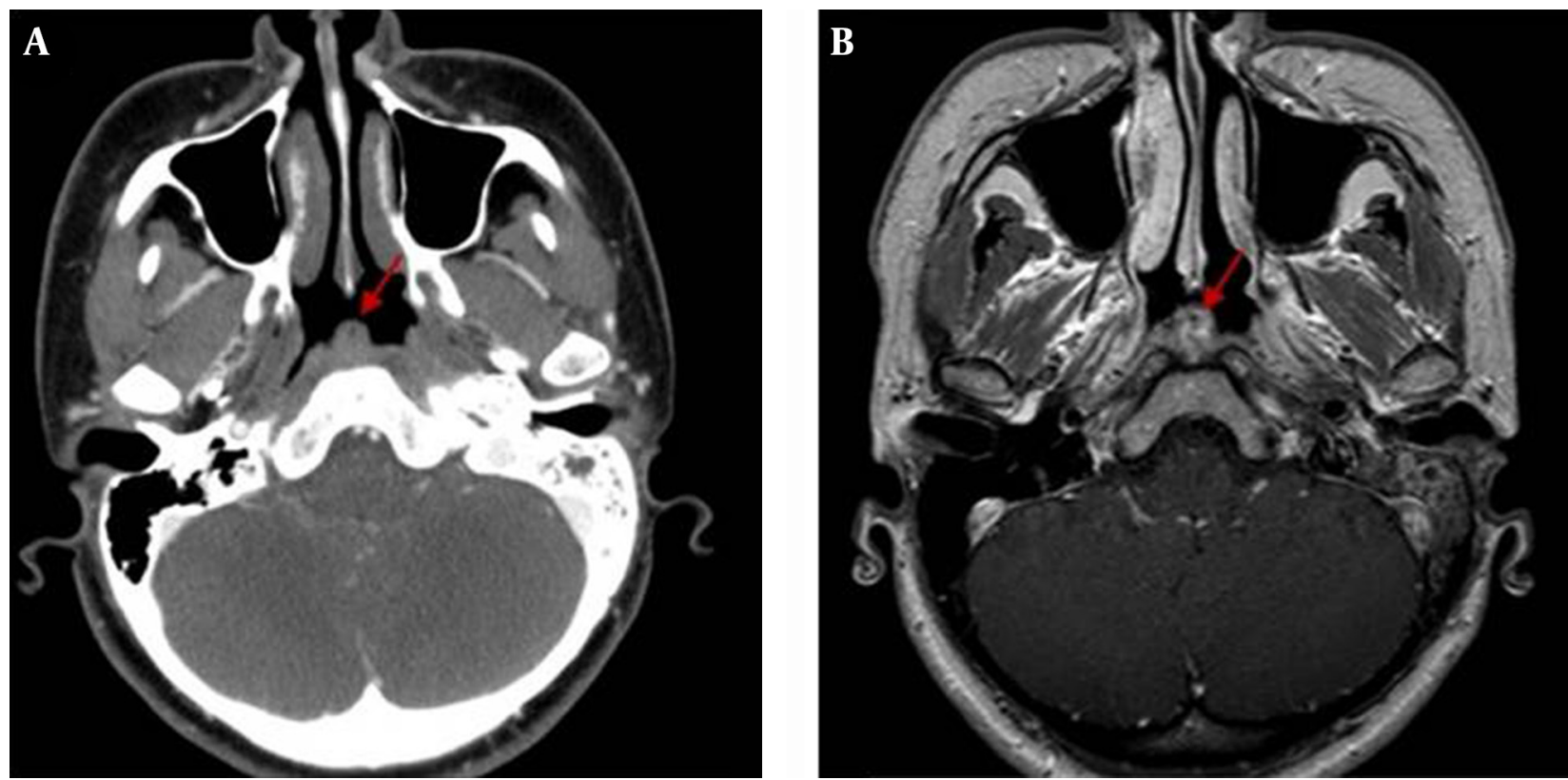

Figure 4. A, Contrast enhanced computed tomography and B, Contrast enhanced T1 weighted magnetic resonance imaging of the paranasal sinuses at the upper nasopharynx show polypoid soft tissue mass (arrows) at posterior roof of the nasopharynx.
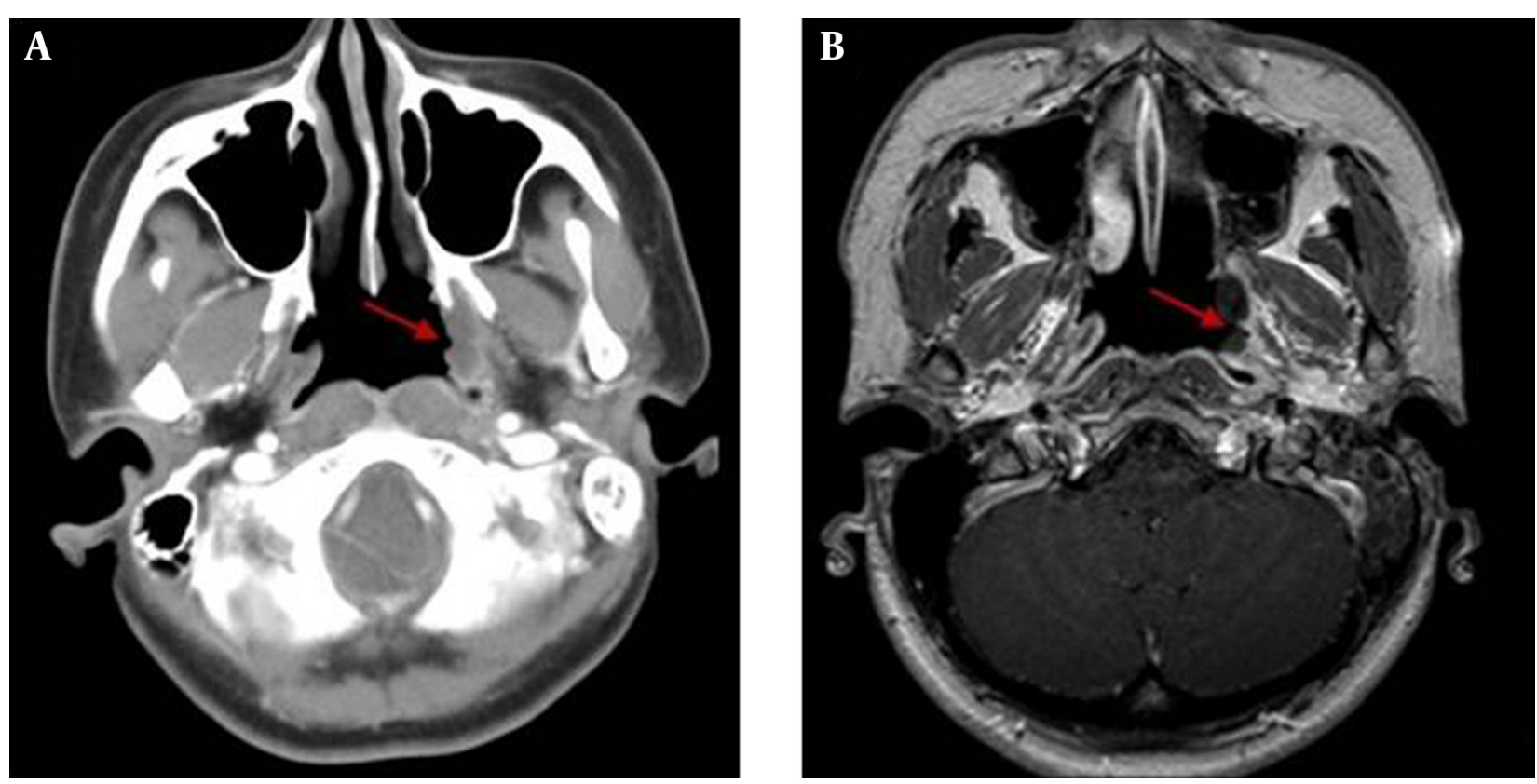

Figure 5. Contrast enhanced tomography and magnetic resonance imaging of the rosenmuller fossa. A, Contrast enhanced computed tomography and $\mathrm{B}$, Contrast enhanced T1 weighted magnetic resonance imaging of the paranasal sinus, at the level of Rosenmuller fossa, show polypoid mass, involving left posterolateral wall of the nasopharynx, with obliteration of the torus tubarius and the pharyngeal opening of Eustachian tube (arrows). 
Histopathology of the biopsy specimen, taken from the nasopharyngeal roof and mastoid antrum confirmed caseating granulomatous inflammation, consistent with TB (Figures 8 and 9). However, the specimen from the nasopharyngeal mass showed negative in AFB staining and polymerase chain reaction (PCR) tests. Considering the low sensitivity of AFB smear and PCR in extrapulmonary specimens, the results from this case report were not unusual. We have reached final diagnosis of primary nasopharyngeal and middle ear TB, on the basis of clinical, radiologic and histopathologic examination, against those from cytology and microbiology examination. The patient was started on four antituberculous medications (rifampicin, isoniazid, pyrazinamide and ethambutol) and underwent left canal wall up (CWU) mastoidectomy, with tympanoplasty. During the operation, multiple polypoid masses were observed in the entire middle ear cavity, mastoid antrum and mastoid air cells. In addition, the pharyngeal opening of the Eustachian tube was obstructed by polypoid masses. All ossicles were surrounded by granulation tissue and an ossiculoplasty was performed. Furthermore, bony canal dehiscence was noted, at the tympanic segment of the facial nerve, and surgical decompression was performed, as well. Histopathology of the biopsy specimens taken from the middle ear cavity and mastoid antrum during the operation also confirmed caseating granulomatous inflammation, consistent with TB. Electrodiagnostic testing, needle electromyogram and nerve conduction studies, were performed three weeks after the operation, which revealed the remaining left facial neuropathy.

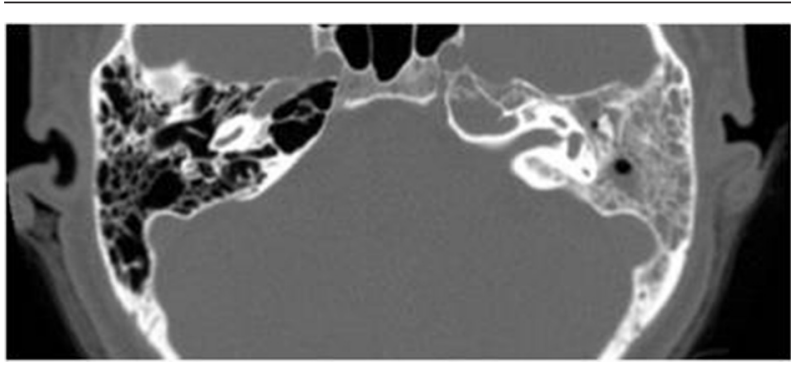

Figure 6. Unenhanced temporal computed tomography. The scan reveals soft tissue attenuation in the entire middle ear cavity, mastoid antrum and the mastoid air cells, without any sclerotic change. Intact ossicles are noted.
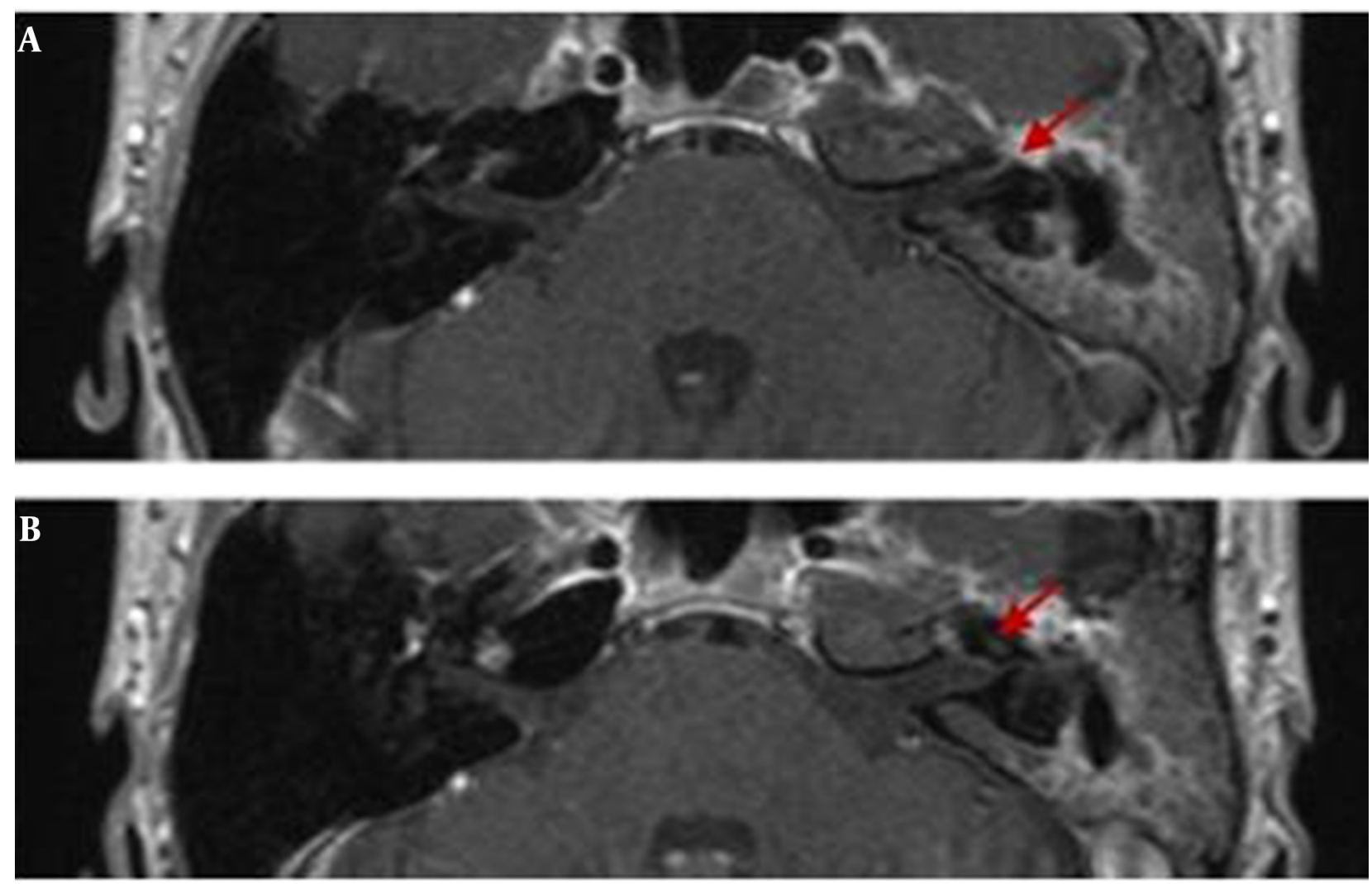

Figure 7. Contrast enhanced T1 weighted magnetic resonance of the seventh nerve. Avid enhancement at the canalicular, genu, labyrinthine, tympanic and mastoid segments of left facial nerve (arrows) are evident. 


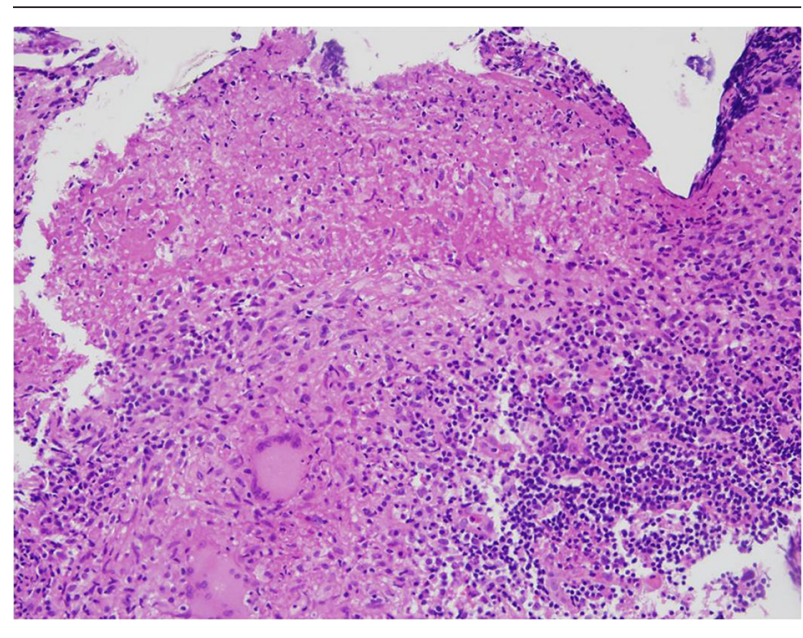

Figure 8. Biopsy of polypoid mass. Histopathologic finding of nasopharyngeal mass shows caseous necrosis and granulomatous inflammation (H\&E stain, $\times 200)$.

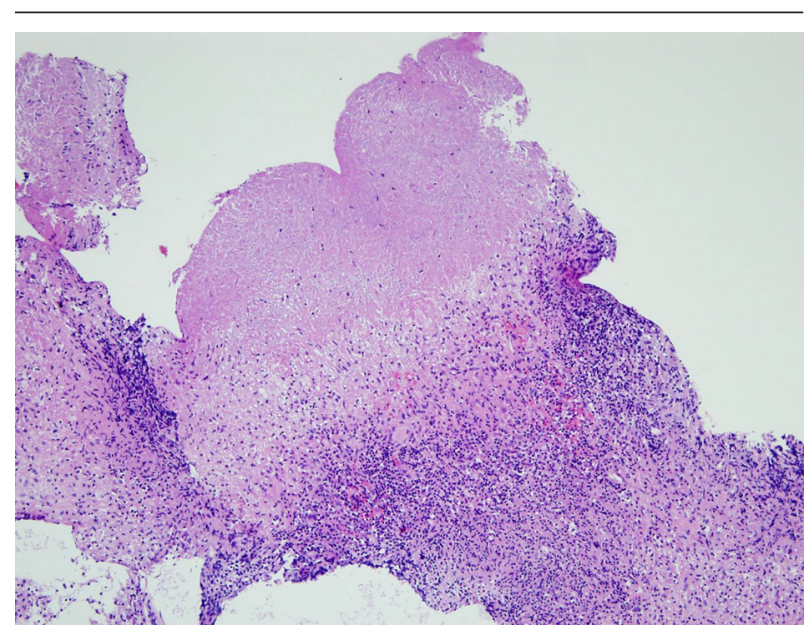

Figure 9. Biopsy of the mastoid antrum. Histopathologic finding of mastoid antrum also shows caseous necrosis and granulomatous inflammation (H \& E stain, $\times 100)$

\section{Discussion}

Upper airway TB is an uncommon clinical condition and is usually combined with pulmonary involvement (1). Moreover, primary TB of the upper respiratory tract, without lung involvement, is rare $(1,2)$. The nasopharynx is the least common site for TB involving the upper respiratory tract and comprises $<1 \%$ of upper respiratory tract TB (4). However, it is interesting to note that recent large scale studies, analyzing nasopharyngeal TB, have reported that primary nasopharyngeal TB is more common than secondary involvement (7). Such discrepancy is probably derived from a limited ability to assess the nasopharynx by physical examination and a low clinical suspicion for nasopharyngeal TB.

Radiographically, nasopharyngeal ТВ presents with two main patterns: 1) polypoid masses and 2) diffuse mucosal thickening $(3,7,8)$. Most cases of nasopharyngeal TB have been identified by a polypoid mass, which has been shown to indicate the proliferative phase (7). The CT and MR imaging show the characteristic findings of nasopharyngeal TB, including presence of necrosis and striped pattern in nasopharyngeal lesions, a lack of invasion of regional structures and peripheral enhancing cervical lymphadenopathy (7). The nasopharyngeal mass or diffuse mucosal thickening may reveal intermediate signal intensity on T1 weighted and T2 weighted images, with moderate contrast enhancement on MR images (8). In the present case, there were also soft tissue densities, in the entire middle ear and mastoid antrum. It has been reported that radiologic characteristics of TOM are soft tissue in the entire middle ear cavity, preservation of mastoid air cells, without any sclerotic change, mucosal thickening of the external auditory canal, with intact scutum (9), which are consistent with our case.

Differential diagnosis can vary, according to radiologic patterns. Lymphoid hyperplasia, nasopharyngeal carcinoma, lymphoma and Castleman's disease should be considered in the differential diagnosis for the polypoid mass. Previous studies have reported that an isolated polypoid mass, with central necrosis centered on nasopharyngeal roof, suggests high probability of nasopharyngeal TB (8). For the second pattern of nasopharyngeal TB, diffuse nasopharyngeal wall thickening, various benign (Wegener's granuloma, syphilis, fungal infection) and malignant lesions (early local stage of nasopharyngeal carcinoma, lymphoma, minor salivary gland tumor) should be considered for the differential diagnosis (8). However, there are no definite imaging features to make an accurate diagnosis of nasopharyngeal TB and a biopsy is required to confirm the diagnosis and to differentiate it from malignancy and the other conditions described above.

It is worth noting that most nasopharyngeal TB involves the posterior roof of the nasopharynx, equivalent to the "adenoid" during childhood or early adolescence (4). The findings lend support to a preceding report, stating that TB directly involves nasopharyngeal lymphoid tissue (3). Regardless, our case showed the two main patterns of primary nasopharyngeal TB: the polypoid mass at the nasopharyngeal roof and, also, the diffuse soft tissue thickening of nasopharyngeal wall, consistent with previous reports and studies. However, the nasopharyngeal carcinoma is most often centered in the lateral pharyngeal recess (also called the Fossa of Rosenmuller). Sites of predilection and morphologic features can be potential diagnostic clues to differentiate nasopharyngeal TB from malignancy.

The Eustachian tube is lined with a mucous membrane, continuous with the pharynx and the mastoid air cells; therefore, the infections from nasopharynx can travel from the nasopharynx along the mucosal membrane of 
the Eustachian tube to the middle ear cavity. In addition, obstructing masses in the nasopharynx prevent air flow from passing through the Eustachian tube, creating a negative pressure in the middle ear cavity, followed by effusion (10). Therefore, it is unclear which came first, the nasopharyngeal TB or TOM, in this case? However, given the physiologic anatomy of the nasopharynx and middle ear cavity, there is a high probability of secondary involvement of the nasopharyngeal TB into the middle ear and mastoid antrum, through pharyngeal opening of the Eustachian tube.

Previous studies of nasopharyngeal TB have not provided in-depth discussion of the possibility for extranasopharyngeal spread and cranial nerve involvement. There have been limited single case reports identifying extensive nasopharyngeal involvement of TB beyond the nasopharyngeal mucosa and submucosa, skull base or parapharyngeal space (1). In this case report, besides in the nasopharyngeal roof, soft tissue masses and mucosal thickening were also noted in the pharyngeal opening of the Eustachian tube. Additional notable findings included soft tissue densities of the ipsilateral middle ear cavity, as well as along the facial nerve canal.

In conclusion, TB infection should be considered in the necrotic polypoid masses with regional mucosal lesions, centered on the posterior nasopharyngeal wall, especially in endemic areas. In addition, radiologists should keep in mind that pharyngeal orifice of the Eustachian tube can be a potential route for the spread of nasopharyngeal $\mathrm{TB}$, from the nasopharynx to the middle ear cavity. Therefore, it is important to determine if obliteration of the nasopharyngeal orifice has occurred, in order to prevent acid bacilli from invading middle ear cavity.

\section{Acknowledgments}

The study design was approved by the appropriate ethics review boards.

\section{Footnotes}

Authors' Contribution:H.Y.Choi analyzed or interpreted the data, drafted and revised the manuscript. E.J.Kim designed or conceptualized the study, analyzed or interpreted the data, and drafted and revised the manuscript. K.M.Lee, J.H.Jang, S.H.Kim, S.G.Yeo and W.S.Choi designed or conceptualized the study.

Financial Disclosure:There are no conflicts of interest to declare.

Funding/Support:None declared.

\section{References}

1. Barman S, Khaund G. Primary tuberculosis of upper airway: case report of 3 rare presentation. Indian J Otolaryngol Head Neck Surg. 2014;66(2):208-11. doi: 10.1007/s12070-012-0536-3. [PubMed: 24822165]

2. Sharma SK, Mohan A. Extrapulmonary tuberculosis. Indian J Med Res. 2004;120(4):316-53. [PubMed: 15520485]

3. King AD, Ahuja AT, Tse GM, van Hasselt AC, Chan AB. MR imaging features of nasopharyngeal tuberculosis: report of three cases and literature review. AJNR Am J Neuroradiol. 2003;24(2):279-82. [PubMed: 12591649]

4. Patil C, Kharat Patil R, Deshmukh P, Biswas J, John B. Primary tuberculosis of nasopharynx (adenoid)- a rare presentation. Asian Pac J Trop Med. 2013;6(3):246-8. doi: 10.1016/S1995-7645(13)600334. [PubMed: 23375043]

5. Vaamonde P, Castro C, Garcia-Soto N, Labella T, Lozano A. Tuberculous otitis media: a significant diagnostic challenge. Otolaryngol Head Neck Surg. 2004;130(6):759-66. doi: 10.1016/j. otohns.2003.12.021. [PubMed: 15195064]

6. Araujo MF, Pinheiro TG, Raymundo IT, Soares VYR, Araujo PIM Melo Martins RL, et al. Tuberculous Otitis Media. Int Adv Otol. 2011;7:413-7.

7. Cai PQ, Li YZ, Zeng RF, Xu JH, Xie CM, Wu YP, et al. Nasopharyngeal tuberculosis: CT and MRI findings in thirty-six patients. Eur J Radiol. 2013;82(9):e448-54. doi: 10.1016/j.ejrad.2013.04.015. [PubMed: 23689055]

8. Prasad BKD, Kejriwal G, Sahu S. Case report: Nasopharyngeal tuberculosis. Indian J Radiol Imaging. 2008;18(1):63-5. doi: 10.4103/0971-3026.38507.

9. Rho MH, Kim DW, Kim SS, Sung YS, Kwon JS, Lee SW. Tuberculous otomastoiditis on high-resolution temporal bone CT: comparison with nontuberculous otomastoiditis with and without cholesteatoma. AJNR Am J Neuroradiol. 2007;28(3):493-6. [PubMed:17353320]

10. Sade J. The nasopharynx, eustachian tube and otitis media. J Laryngol Otol. 1994;108(02):95-100. doi: 10.1017| s0022215100126003.[PubMed: 7802761] 